\title{
Athermal All-Optical Femtosecond Magnetization Reversal in GdFeCo
}

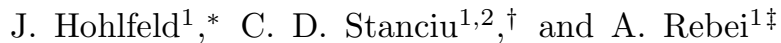 \\ 1. Seagate Research Center, Pittsburgh, PA 15222 \\ 2. Institute for Molecules and Materials, Radboud University Nijmegen, \\ Toernooiveld 1, 6525 ED Nijmegen, The Netherlands
}

(Dated: October 29, 2018)

\begin{abstract}
Magnetization reversal in GdFeCo by circularly polarized light is shown to occur at the femtosecond time scale. In contrast to the well known laser-assisted magnetization reversal based on the laser heating, we here demonstrate that this femtosecond all-optical magnetization reversal is more efficient at lower temperatures. The lower the temperatures, the smaller the laser fluence required for the switching. This switching is in agreement with a more recent theoretical prediction [Phys. Lett. A 372, 1915 (2008)] and demonstrates the feasibility of the femtosecond athermal magnetization reversal.

PACS numbers: 76.60.+q,78.20.Ls,75.40.Gb, 76.60.Es, 52.38.-r
\end{abstract}

The current development in electronic devices demands increasingly fast approaches to manipulate logical bits. In a magnetic-memory device the speed of this process relies on fast switching of the magnetization vector. Currently, the switching of a magnetic bit in a hard disk drive occurs as quickly as $500 \mathrm{ps}$, via the application of an external magnetic field. A possible much faster approach for the magnetization reversal has been indicated a decade ago, with the demonstration of the laser induced ultrafast demagnetization in magnetic metals [1]. Yet, this phenomena involved only the breakdown of the magnetization vector, via heating, but not its control.

Employing 40 femtosecond circularly polarized laser pulses it has been recently demonstrated that laser alone can be used to control the direction of the magnetization [2]. It was shown that the reversal of the magnetization can be controlled by the helicity of the light and no external magnetic field is required. This new magnetization reversal mechanism was understood as the combined result of femtosecond laser heating of the magnetic system to just below the Curie point and circularly polarized light simultaneously acting as a magnetic field. On the other hand, it has been argued recently that this magnetization reversal is completely athermal, and therefore does not require heating near Curie temperature [3]. More specifically, it was proposed that for suitable parameters of the coupling between the $d$ spins and the $f$ spins of the rare-earth, and in the presence of a strong laser-enhanced spin-orbit coupling of $d$ electrons (such as recently demonstrated in ferromagnetic $\mathrm{Ni}$ [4]), femtosecond athermal switching is possible in rare-earth doped transition metals, via an inverse Einstein-De Haas effect (i.e. Barnett effect [5]). A microscopic theory on the laser-induced magnetization in a metallic material, to some extend similar to the Barnett effect, has been also recently proposed [6]. Nevertheless, the mechanisms responsible for the all-optical permanent magnetization reversal in metals are currently a matter of debate. Besides this, another open question is related to the all- optical switching reversal speed. In Ref. [2] it is shown that each circularly polarized 40 fs laser pulse leads to the formation of a permanent magnetic domain. But what is the timescale of this reversal?

In this letter, using a time resolved pump-probe set-up, we investigate the reversal time of all-optical switching. It is experimentally demonstrated that the reversal occurs in the sub-picosecond regime. In agreement with a very recent observation [4], this sub-picosecond switching rate indicates that relaxation effects induced by optical excitations are much larger than those derived from ferromagnetic resonance measurements. In addition, by investigating the temperature dependence of the all-optical switching we show that this process is taking place even at low temperatures. More specifically, the lower the temperatures, the smaller the laser fluence required for the switching. This observation demonstrates the pure athermal origin of the reversal mechanism.

The time resolved experiments where performed in Pittsburgh, employing relatively long laser pulses, of $\approx 500$ fs duration, while the temperature dependent measurements were carried out in Nijmegen using 40 fs laser pulses. The metallic magnet studied here was an amorphous GdFeCo ferrimagnetic alloy, with the same composition as in Ref. [2].

We first start by discussing the time resolved measurements. The corresponding set up is shown in Fig. 1. The pump-probe pulses use a two-color scheme with a wavelength of $\lambda=800 \mathrm{~nm}$ pump-, and $\lambda=400 \mathrm{~nm}$ probepulses. The pump-pulses of $100 \mu \mathrm{J}$ energy are focused at normal incidence onto the sample, via a $700 \mathrm{~mm}$ lens. The collinear probe-pulses are attenuated to $1 \mu \mathrm{J}$ to avoid significant self-action, and are focused with the help of a $100 \mathrm{~mm}$ lens. The diameter of the focused pump- and probe-beams was estimated to be of the order of $500 \mu \mathrm{m}$ and $50 \mu \mathrm{m}$, respectively. The Kerr-rotation of the probepulses was recorded by a standard two diode scheme [7] and lock-in detection using the $500 \mathrm{~Hz}$ repetition rate of the laser system as a reference frequency. The sample 
(disk) is mounted on a fast rotation stage which ensures that every pump-probe pulse-pair is exciting/probing a new spot initialized by a permanent magnet mounted near the sample (see Fig. 1).

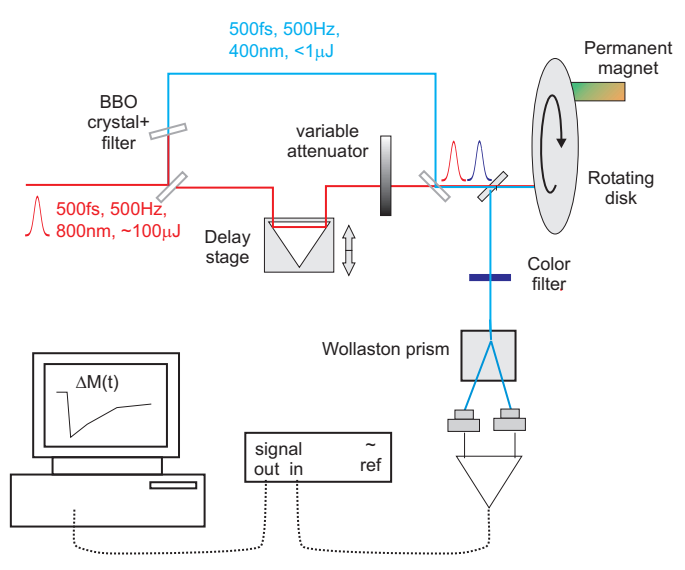

FIG. 1: Schematic drawing of the time-resolved pump-probe experimental set-up used to measure the dynamics of alloptical switching on the femtosecond time scale.

The pump pulse duration was determined by comparing measured and calculated second-order intensity correlation functions as shown in Fig. 2. The best fits for pulse shapes of the form of a Gaussian and of a $\operatorname{sech}^{2}$ lead to actual pulse lengths of $570 \mathrm{fs}$, and $450 \mathrm{fs}$, respectively. Hence, we assume the pulses to be of $\approx 500 \mathrm{fs}$ duration, one order of magnitude larger than the Nijmegen laser pulse width.

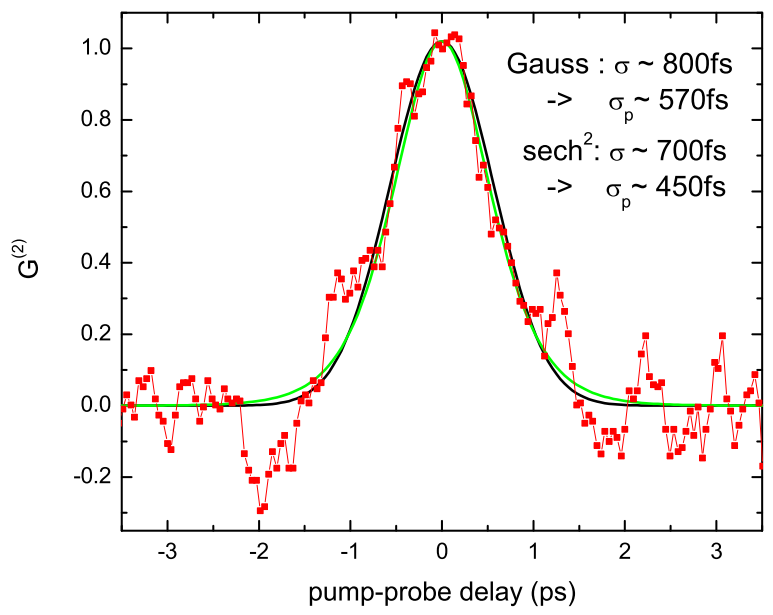

FIG. 2: Second order intensity correlation, $G^{(2)}$, as measured for the laser pulse used in the real-time measurements (filled squares). The continuous lines represent best fits to Gaussian and sech ${ }^{2}$ pulse shapes which both give similar pulse widths.

Using relatively long pump-pulses, the optically excited electrons have lots of time to thermalize among

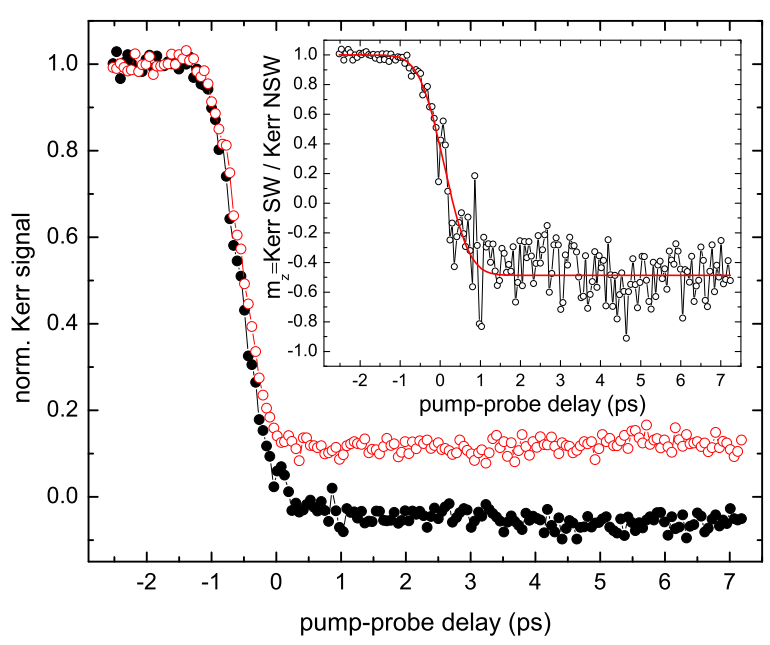

FIG. 3: Transient magnetization dynamics induced by circularly polarized pump-pulses with helicity favoring the initial orientation of the magnetization (NSW, open symbols) and leading to magnetization reversal (SW, closed symbols). The inset shows the resulting dynamics of the normalized out-of plane component of the magnetization, $m_{z}$, obtained by dividing the transient for the switching case by the one for the non-switching configuration. The line is to guide the eye and represents an error-function of $700 \mathrm{fs}$ width.

each other and even to transfer a significant amount of energy to the lattice while the pump-pulse is on. Hence, we observe a significant reduction of the magnetization magnitude during the reversal process (see Fig. 3). This apparent disadvantage is more than compensated by the successful demonstration of all-optical switching with $\approx 500 \mathrm{fs}$ pulses that are far closer to real applications than the more exotic $40 \mathrm{fs}$ pulses used previously [2].

In order to separate switching dynamics from purely temperature induced changes in the magnitude of the magnetization, we use a procedure that was successfully applied to analyze the dynamics of magneto-optical recording [8]. Here, we use the normalized out-of plane component of the magnetization, $m_{z}$, derived from the ratio of the Kerr signals measured for both pump helicities, i.e. for the switching and non-switching cases, to extract the switching dynamics. It is clear from the time dependence of $m_{z}$, shown in the inset of Fig. 3, that the switching is very fast and occurs within one picosecond. For our laser pulse of width $500 \mathrm{fs}$, we estimate that the switching is complete within 700 fs. The switching is therefore too fast to follow a precessional path. Indeed, the model discussed in Ref. [3] shows that precessional dynamics is not important for femtosecond magnetization reversal. Figure 3 also shows that our measurements yield a minimum value of $m_{z}=-0.5$ instead of -1.0 , thereby indicating that on average only $75 \%$ of the pump pulses lead to a reversed state. Because the all-optical switching is strongly sensitive to a change in 
the pump fluence [2], a complete $100 \%$ reversal requires a very fine tuning of the laser fluence, which was difficult to attain in our experiments. Shorter laser pulses should yield stable switching over a wider fluence range and be able to diminish the effect of heat on the magnetization.

To explain this femtosecond magnetization reversal by circularly polarized light, three main requirements must be fulfilled: 1) an ultrafast channel for angular momentum exchange, between the spins and another degree of freedom, such as lattice; 2) a light induced switching mechanism, where the magnetization direction is controlled by the light helicity; 3) a mechanism that, after switching, maintains information about light helicity in spite of the decoherence effects that takes place in metallic magnets on the femtosecond time scale. We will now briefly discuss each of these mechanisms:

1) We here observe an all-optical magnetization reversal taking place on the femtosecond time scale. Therefore, it implies an ultrafast transfer of angular momentum from the spin system to another degree of freedom such as the orbital momentum of electrons or lattice. In turn this also implies a strong spin-orbit interaction during the optical excitation. Such fast coupling is in agreement with the recent experimental demonstration of a laser enhanced spin-orbit coupling in $\mathrm{Ni}$ [4]. Hence we believe, as also discussed in Ref. [3], that relaxation of the non-equilibrium $d$ electrons plays an important role in increasing the number of channels available to relax the magnetization through spin-orbit coupling or momentum relaxation [9]. A sub-picosecond switching rate also indicates that relaxation effects induced by optical excitations are much larger than those derived from ferromagnetic resonance measurements (FMR). This is in line with the different energy scales of $1 \mathrm{eV}$ and $1 \mathrm{meV}$ for optical switching and FMR, respectively.

2) How is it possible that the angular momentum of the photons can efficiently change magnetization? The optical electric dipole transition can not affect the electronic spin. Magnetic dipole transition may affect the spin but it requires annihilation of the photon. However, there are not enough photons in the laser pulse to provide enough angular momentum for a magnetization reversal [10]. An efficient switching mechanism may take place via a stimulated Raman-like scattering process [7]. Yet, this switching mechanism requires heating of the spin system to temperatures close to Curie temperature [2]. As we will further demonstrate, the all-optical switching takes place at a temperature of $200 \mathrm{~K}$ lower than that used in Ref. [2]. Based on this result, a more realistic scenario may be accounted to an optical Barnett effect which works best at zero temeprature [3].

3) Another issue that needs to be clarified is how the femtosecond helicity-induced coherence among the itinerant electrons may be converted to a static magnetization. It is well known that the lifetime of a state at $1 \mathrm{eV}$ above the Fermi level is a few femtoseconds [11]. Relaxation of these excited states leads to the destruction of the spin coherence via inelastic scattering processes. Indeed, it is by now well accepted that there is no helicity-induced magnetization reversal in pure transition-metals such as $\mathrm{Ni}$, under similar conditions [12]. Thus, the observed memory effect in our experiments may be explained as follows: During the optical excitation, the negative exchange between the excited $d$ electrons of both Gd and Fe collapses [13]. On the other hand, the rare-earth $4 f$ bands are about $4 \mathrm{eV}$ below the Fermi level and are therefore not directly excited by the $1.5 \mathrm{eV}$ photon energy used in our experiments. Yet, a strong hybridization between the $d$-spins and the $f$-spins of the rare-earth causes the component of the $f$-spins along the chirality of the laser to change sign at high enough laser powers. Therefore, although the spin coherence of the excited states may be lost via relaxation in electron-electron scattering, $f$-spins will maintain sufficient coherence in the reversal process and therefore maintain information about the chirality of the laser. In this way, $f$-spins will serve as nucleation points for what will later become, via proceses such as domain wall propagation, a complete reversed magnetic domain. This explains the importance of the rare earth moments. A convincing test of this model will be the investigation of the all-optical switching in $\mathrm{SmCo}_{5}$ and $\mathrm{YCO}_{5}$. The former has uncompensated $f$-spins, but the latter does not while both have similar crystal structure and magneto-crystalline anisotropy.

As discussed above, an important factor indicating whether the reversal mechanism takes place via a stimulated Raman-like scattering or via an optical Barnet effect, is the relevance of temperature in the reversal process. We therefore turn our attention towards the low temperature all-optical switching experiments.

The temperature dependence of the all-optical switching has been performed in a $\mathrm{Gd}_{22} \mathrm{Fe}_{74.6} \mathrm{Co}_{3.4}$ film with a $20 \mathrm{~nm}$ thickness. The sample structure was described in Ref. [2]. The sample has been placed into a cryostat and exposed to $40 \mathrm{fs}$ circularly polarized laser pulses generated at a repetition rate of $1 \mathrm{kHz}$. The laser wavelength was $800 \mathrm{~nm}$ and the laser helicity has been chosen to be that relevant for the switching. Initially, at $300 \mathrm{~K}$, in order to indicate the location of the laser spot onto the sample, $\mathrm{GdFeCo}$ has been exposed to a laser fluence of about $3 \mathrm{~mJ} / \mathrm{cm}^{2}$, for a few seconds. Next, the laser fluence has been reduced to about $2.5 \mathrm{~mJ} / \mathrm{cm}^{2}$, that is below the fluence threshold required for the all-optical switching in this sample 2]. At this laser fluence no switching could be observed. Under these conditions the temperature on the sample was reduced down to $10 \mathrm{~K}$ at a rate of about $5 \mathrm{~K}$ per minute while the sample was slowly vertically shifted (from down to up). One can observe in Fig. 4, that while from $300 \mathrm{~K}$ down to $250 \mathrm{~K}$ the laser fluence of $2.5 \mathrm{~mJ} / \mathrm{cm}^{2}$ does not change the magnetic state of the 


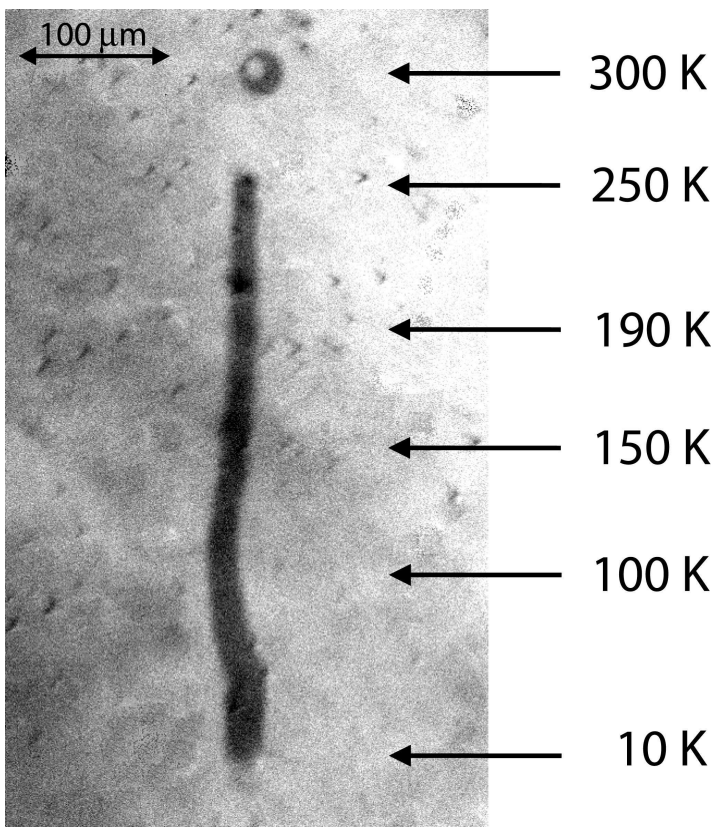

FIG. 4: All-optical switching of magnetization as a function of temperature, in GdFeCo. Black and white areas correspond to oppositely oriented magnetic domains, perpendicular to the sample plain. The dot-like magnetic domain was created in order to indicated the laser beam location onto the sample, at $300 \mathrm{~K}$. The switching behavior as a function of temperature has been investigated at a laser fluence of $2.5 \mathrm{~mJ} / \mathrm{cm}^{2}$. At this laser fluence no switching is observed at room temperature but it becomes visible only when the temperature is reduced below $250 \mathrm{~K}$.

sample, the conditions change drastically below $250 \mathrm{~K}$. More specifically, below this temperature the switching spot appears and increasingly broadens as the temperature is reduced down to $10 \mathrm{~K}$. This observation gives clear evidence that thermal fluctuations introduce decoherence in the system that upsets the reversal process. Hence increasing the temperature decreases the efficiency of the all-optical switching.

It has been initially argued that besides the nonthermal effect where the light acts as an axial magnetic field, the heat from the thermal bath is also required to elevate the temperature of the magnetic system near Curie temperature 2]. The authors in [3] argued and qualitatively showed that switching is possible even at zero temperature if we take into account the non-equilibrium character of the laser-induced process. The temperature dependent data presented here clearly favor the latter suggestion. Note that the magnetization switching path at zero temperature is very similar to switching by a strong field at the Curie temperature [14] but the physics in both cases is very different. The Barnett effect discussed in [3] induces a frequency dependent interaction between the rare-earth ions and the itinerant $d$ electrons of the transition metal ions that, depending on the helicity of the light, gives rise to either a ferromagnetic or anti-ferromagnetic coupling.

In conlcusion, we have demonstrated that all optical switching in GdFeCo is not a thermally assisted process but in fact thermal fluctuations degrade the efficiency of the reversal. Moreover, we showed that the switching occurs on a sub-picosecond time scale which can not be explained in terms of the much slower precessional reversal. Both findings, i.e. fast and athermal mechanism, make all-optical recording on rare-earth transition metal alloys a promising technique for magnetic storage with high data rates. Furthermore, the all-optical switching using the long 500fs laser pulses shown here, together with the recent successful demonstration of miniature plasmonic wave-plates [15], push the all-optical switching close to applications.

One of the authors (C. D. S.) expresses his gratitude to Prof. Rasing for mentorship. We also thank Prof. A. Tsukamoto for preapring the samples used in this study and Dr. Paul Jones for assistance with the laser set up. A.R acknowledge fruitful discussions with Prof. Roy Chantrell regarding his temperature calculations in similar problems.

* Electronic address: julius.hohlfeld@seagate.com

$\dagger$ Electronic address: daniel@stanciu.nl

‡ Electronic address: arebei@mailaps.org

[1] E. Beaurepaire et al., Phys. Rev. Lett. 76, 4250 (1996).

[2] C. D. Stanciu, F. Hansteen, A. V. Kimel, A. Kirilyuk, A. Tsukamoto, A. Itoh, and Th. Rasing, Phys. Rev. Lett. 99, 047601 (2007).

[3] A. Rebei and J. Hohlfeld, Phys. Lett. A 372, 1915 (2008); A. Rebei and J. Hohlfeld, J. Appl. Phys. 103, 07B118 (2008).

[4] C. Stamm, T. Kachel, N. Pontius, R. Mitzner, T. Quast, K. Holldack, S. Khan, C. Lupulescu, E. F. Aziz, M. Wietstruk, H. A. Durr, W. Eberhardt, Nature Mat. 6, 740 (2007).

[5] S. J. Barnett, Phys. Rev. 6, 239 (1915).

[6] R. Hertel, J. Magn. Magn. Mater. 303, L1 (2006).

[7] C. D. Stanciu, Ph.D thesis, Nijmegen, The Netherlands 2008.

[8] J. Hohlfeld, Th Gerrits, M. Bilderbeek, and Th. Rasing, Phys. Rev. B 65012413 (2002).

[9] A. Rebei and J. Hohlfeld, Phys. Rev. Lett. 97, 117601 (2006).

[10] B. Koopmans, M. van Kampen, J.T. Kohlhepp, W. J. M. de Jonge, Phys. Rev. Lett. 85, 844 (2000).

[11] M. Aeschlimann, M. Bauer, S. Pawlik, W. Weber, R. Burgermeister, D. Oberli, and H. C. Siegmann, Phys. Rev. Lett. 79, 5158 (1997).

[12] F. Dalla Longa, J. T. Kohlhepp, W. J. M. de Jonge, and B. Koopmans, Phys. Rev. B 75, 224431 (2007).

[13] H.-S. Rhie, H. A. Dürr, and W. Eberhardt, Phys. Rev. Lett. 90, 247201 (2003).

[14] R. W. Chantrell, private communication.

[15] Aurélien Drezet, Cyriaque Genet, and Thomas W. Ebbesen, Phys. Rev. Lett. 101, 043902 (2008). 